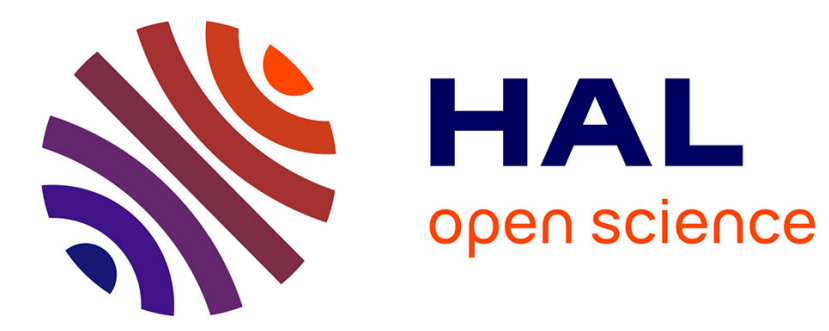

\title{
2D Landmark-Based Facial Asymmetry Assessment in the Clinical Case of Facial Paralysis
}

Benjamin Szczapa, Mohamed Daoudi, Anis Kacem, Pierre Guerreschi, Ludwig Gebert, Juan Carlos Alvarez-Paiva

\section{- To cite this version:}

Benjamin Szczapa, Mohamed Daoudi, Anis Kacem, Pierre Guerreschi, Ludwig Gebert, et al.. 2D Landmark-Based Facial Asymmetry Assessment in the Clinical Case of Facial Paralysis. The 14th IEEE International Conference on Automatic Face and Gesture Recognition (FG 2019), May 2019, Lille, France. hal-02371137

\section{HAL Id: hal-02371137 https://hal.science/hal-02371137}

Submitted on 19 Nov 2019

HAL is a multi-disciplinary open access archive for the deposit and dissemination of scientific research documents, whether they are published or not. The documents may come from teaching and research institutions in France or abroad, or from public or private research centers.
L'archive ouverte pluridisciplinaire HAL, est destinée au dépôt et à la diffusion de documents scientifiques de niveau recherche, publiés ou non, émanant des établissements d'enseignement et de recherche français ou étrangers, des laboratoires publics ou privés. 


\title{
2D Landmark-Based Facial Asymmetry Assessment in the Clinical Case of Facial Paralysis
}

\author{
Benjamin Szczapa ${ }^{1}$, Mohamed Daoudi ${ }^{1}$, Anis Kacem ${ }^{1}$, Pierre Guerreschi ${ }^{3}$, Ludwig \\ Gebert $^{4}$, Juan Carlos Alvarez-Paiva ${ }^{2}$ \\ ${ }^{1}$ IMT Lille Douai, Univ. Lille, CNRS, UMR 9189 CRIStAL Centre de Recherche en Informatique Signal et \\ Automatique de Lille, F-59000 Lille, France \\ ${ }^{2}$ University of Lille and Painlevé laboratory, CNRS-UMR-8524, France \\ 3 Service de chirurgie plastique reconstructrice et esthétique, Université of Lille \\ ${ }^{4}$ Service de chirurgie plastique, reconstructrice et esthétique du Centre Hospitalier Saint-Joseph Saint-Luc, 20 \\ quai Claude Bernard, 69007 Lyon
}

\begin{abstract}
In this paper, we propose a novel technique for quantifying the facial asymmetry from $2 \mathrm{D}$ videos to evaluate facial paralysis treatments based on Botulinum Toxin (BT) injections. Our approach uses 2D facial landmarks and barycentric coordinates to objectively quantify the facial asymmetry across 2D videos. To assess our approach, a new dataset of $2 \mathrm{D}$ videos, containing eighteen patients before and after the treatments have been collected. For each patient, we have collected nine facial expressions. Experimental results on the newly collected dataset show that the proposed approach provides promising results in concordance with clinical annotations.
\end{abstract}

\section{INTRODUCTION}

The facial asymmetry can be seen in patients with facial paralysis whose etiologies may be multiple (infectious, tumoral, traumatic, iatrogenic, inflammatory, vascular, congenital, idiopathic). A common medical treatment, widely used since 1989 in surgery and aesthetic medication [1], [3], involves injecting into the facial muscles low doses of botulinum toxin (BT) to improve harmony and facial symmetry. For facial palsy, the role of BT treatment will be to reduce the spasm and synkinesis of the paralyzed hemiface and the muscular hyperactivity of the healthy hemiface of the face. In general, the results of the medical treatments are evaluated subjectively by the clinicians after few weeks of each BT injection, using medical scales. The two most commonly used scales are the House-Brackmann scale [8] and the Sunnybrook facial grading system [13]. With these scales, the clinicians can evaluate different part of the patient's face and define a score based on patient's asymmetry. Nonetheless, there is no standard scale to define the asymmetry of the face today while there is a strong need for the clinicians to have objective measures. However, with the increase of image systems, new techniques were developed by analyzing 2D or 3D images. For instance, Desrosiers et al. [2] proposed a method to compute the asymmetry degree of paralyzed persons captured with a 3D scanner. Their approach was based on the use of 3D meshes to compare the paralyzed side of the face with the healthy side. Even, this approach gave promising results, the use of 3D scanner and accompanying software make this technique expensive in cost and time. Taking another direction, Gaber et al. [4], [5] presented a proof of concept using a more cost effective 3D sensor (i.e., Microsoft Kinect) to compute the facial asymmetry based on a tracked Active Appearance Model (AAM), but only tested it on healthy subjects. Gerós et al. [6] used RGB-D cameras to track 3D facial landmarks marked with ink dots on the patient's face. They also performed a head movement correction by placing two points on the forehead, blocking the asymmetry analysis on this region. Horta et al. [7] proposed a semiautomatic method to evaluate the asymmetry of $2 \mathrm{D}$ facial images. However, it requires the user initialization. In [11], a smartphone based system was proposed to evaluate the asymmetry but it is no clear how this approach is robust to the distortions due to patient's head movements.

In this paper, we propose a novel approach based on the barycentric coordinates of automatically tracked 2D facial landmarks from videos to objectively evaluate the facial asymmetry. For each frame of a 2D face sequence, 2D facial landmarks of the face are detected using a state-of-the-art solution. The barycentric coordinates [9], [10] are computed from the 2D landmarks to make our approach robust to affine transformations and reduce the noise due to patient's head movements. With the barycentric coordinates, we can perform two simple operations to get the symmetric and the theoretical symmetric of the landmarks. By comparing these two operations, we obtain an asymmetry index indicating the degree of the facial asymmetry in this frame. This technique quantifies the evolution of the asymmetry index across the 2D sequences. An overview of our approach is shown in Fig. 1. The main contributions of this paper are: 1) A novel technique to quantify objectively and dynamically the facial asymmetry before and after BT injections, 2) A new dataset of patients with facial paralysis that uses different expressions to compare our work with the medical expertise. 


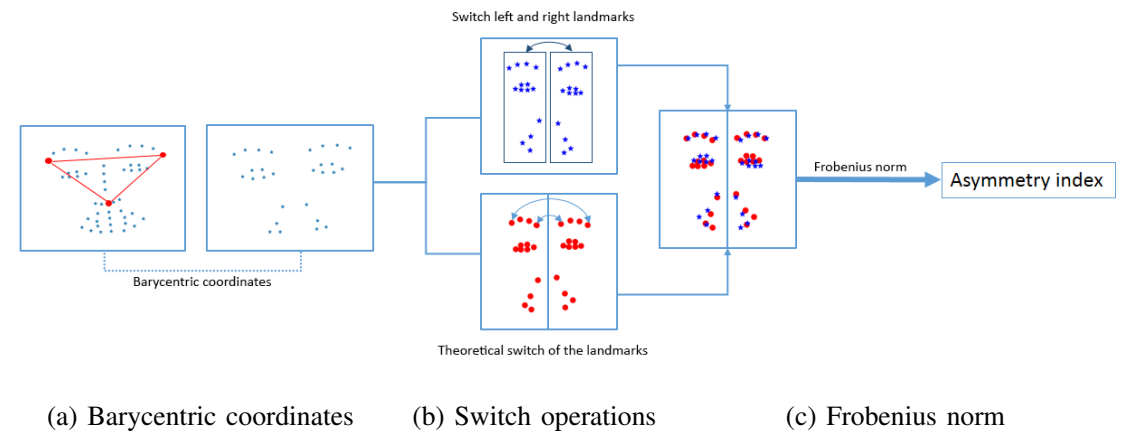

Fig. 1: Approach overview. (a) Computation of the barycentric coordinates for the detected 2D landmarks. (b) Switch of the right hemiface and left hemiface landmarks and theoretical switch of the landmakrs. (c) Computation of the asymmetry index by computing the Frobenius distance between the two switches.

\section{OUR APPROACH}

Given a facial image, the first step to our approach is detecting a set of two-dimensional fiducial points on relevant regions of the face (e.g., eyes, eyebrows, mouth, and nose). Accordingly, we use Intraface [12] to detect 49 points around these regions.

\section{A. Barycentric Coordinates}

One common problem encountered in many facial analysis tasks is the view variations of the face with respect to the camera. These variations are accentuated by the head pose changes and result in undesirable transformations that should be filtered out in order to conduct a view-invariant analysis of the face. In the context of facial landmarks, the barycentric coordinates have shown promising results in doing so [9], [10]. This method requires the choice of three points among the facial landmarks forming a non-degenerate triangle, and results in an affine-invariant representation of the 2D facial landmarks. More precisely, let us consider a facial landmark configuration $L$ consisting of $N$ two-dimensional points $L_{1}=\left(x_{1}, y_{1}\right), L_{2}=\left(x_{2}, y_{2}\right), \ldots, L_{N}=\left(x_{N}, y_{N}\right)$. As stated in [9], the first step to build the barycentric coordinates of $L$ consists of passing to the homogeneous coordinates of the landmark points by adding a nonzero constant $\alpha$ as a third coordinate for the landmark points. Assuming that $L_{1}, L_{2}, L_{3}$ form a non-degenerate triangle, the $(N-3) \times 3$ matrix

$$
\Lambda=\left(\begin{array}{ccc}
x_{4} & y_{4} & \alpha \\
\vdots & \vdots & \vdots \\
x_{N} & y_{N} & \alpha
\end{array}\right)\left(\begin{array}{ccc}
x_{1} & y_{1} & \alpha \\
x_{2} & y_{2} & \alpha \\
x_{3} & y_{3} & \alpha
\end{array}\right)^{-1}
$$

consists of the barycentric representation of the landmark configuration $L$ and is invariant to affine transformations of the plane. In theory the choice of $\alpha$ is irrelevant, although for numerical stability it is not convenient to choose it too close to zero. In practice, we set the value of $\alpha$ to 100 and chose the point under the nose tip (i.e., philtrum) and the corners of left/right eyebrows to define the non-degenerate triangle. In what follows, we will consider the $n \times 3$ matrix $\Lambda$ with
$n=N-3$ to represent a $2 \mathrm{D}$ facial landmark configuration of $N$ points and $\lambda_{i}, 1 \leq i \leq n$, to denote the barycentric coordinates of a landmark point.

\section{B. Global Asymmetry Index}

We seek an objective way to measure the facial asymmetry from facial images based on the barycentric representation of the extracted 2D facial landmarks introduced in the previous section. To do so, we exploit the natural symmetry of the human face and assume that the right and left halves of the face are each of them composed of $m=n / 2$ barycentric coordinates. We assume further that these coordinates are ordered in the matrix $\Lambda$ in such way that $\lambda_{i}$ and $\lambda_{i+m}$ correspond to symmetric points on the face. There are two simple operations that we can perform on $\Lambda$ to quantify the asymmetry of the face.

On one hand, we can multiply

$$
S \Lambda=\left(\begin{array}{cc}
0 & I_{m} \\
I_{m} & 0
\end{array}\right) \Lambda,
$$

where $I_{m}$ is the $m \times m$ identity matrix. This corresponds to switching the right and the left landmarks of the face.

On the other hand, we can multiply

$$
\Lambda T=\Lambda\left(\begin{array}{lll}
0 & 1 & 0 \\
1 & 0 & 0 \\
0 & 0 & 1
\end{array}\right)
$$

which corresponds to the theoretical switch of the right and left landmarks, provided the symmetry we are currently studying. Finally, we can compute the square of the Frobenius norm of the difference between $S \Lambda$ and $\Lambda T$ to get the asymmetry index

$$
\begin{aligned}
\text { Ind } & =\|S \Lambda-\Lambda T\|_{F}^{2} \\
& =\operatorname{Tr}\left[(S \Lambda-\Lambda T) *\left(\Lambda^{\top} S-T \Lambda^{\top}\right)\right] \\
& =2 * \operatorname{Tr}\left(\Lambda \Lambda^{\top}\right)-2 * \operatorname{Tr}\left(S \Lambda T \Lambda^{\top}\right),
\end{aligned}
$$

where $\|.\|_{F}$ denotes the Forbenuis norm and $\mathrm{Tr}$ is the trace of a matrix. In this computation we have made use that $S$ and $T$ are symmetric matrices and that each is its own inverse. 
The result of Eq. 4 is the asymmetry index, with respect to the symmetry of the face, for one image. This operation is performed on each frame of the facial image sequences. Note that we do not use all the facial landmarks provided by Intraface detector [12]. In fact, several landmarks are close to the axis of symmetry of the face and therefore can be present in different sides (left and right) of the face. These points are not considered in our study. In the end, we use 28 landmark points, 14 points on each hemifaces.

\section{Local Asymmetry Index}

The global asymmetry index introduced in the previous section assigns an asymmetry index for the whole face. However, it does not inform on the most asymmetric local regions of the face. To evaluate locally the asymmetry of the face, we compute three local indices for three regions (i.e., eyes, eyebrows, and mouth) instead of computing a global asymmetry index. To do so, we extract from the global representation $\Lambda$ the barycentric coordinates of each region $\Lambda_{R}^{j}, 1 \leq j \leq 3$, and compute its local asymmetry index $\operatorname{Ind}_{j}$ according to Eq. 4. In the context of facial paralysis, these local asymmetry indices can also help to detect involuntary muscular movements that can happen when a patient is conveying an expression [3]. For instance, they can detect an involuntary eye closure in a patient's smile. The detection of these involuntary movements in local regions is crucial to clinicians since BT injections should be mainly performed on these local regions of the face.

\section{EXPERIMENTAL RESULTS}

\section{A. Dataset collection}

We collected a dataset of 18 patients suffering from facial paralysis (three men and fifteen women), aged from 29 to 85 , with an average age of 53. Nine of these patients have their right hemiface paralyzed and the other nine have their left hemiface paralyzed. We also recorded eight healthy subjects (three men and five women), aged from 24 to 42 , with an average age of 28 . These healthy subjects were recorded to have a baseline for the asymmetry that we are studying. Each patient was recorded in two sessions (before and after treatments). During each session, the subjects were asked, after agreement, to seat on a chair in front of the camera and to perform eight facial expressions and pronounce a sentence. Table I summarizes the expressions that were asked to the patients. These expressions were chosen because they activate special face muscles allowing the clinicians to effectively evaluate the facial asymmetry of the patients with the available medical scales. Accordingly, each session, which consists of nine video sequences of a patient, was annotated by clinicians according to two different medical scales namely, House-Brackmann [8] and Sunnybrook [13] scales. In total, we recorded 324 video sequences of the patients, resulting in 14091 images. To facilitate the comparison of the sequences before and after the treatments, we normalized the video sequences by only keeping the frames where the subject is performing the facial expression. After normalization, we obtained a total of 9044
TABLE I: Types of expressions during a session

\begin{tabular}{l|l} 
ID & Signification \\
\hline E1 & Neutral expression \\
E2 & Big smile \\
E3 & Normal smile \\
E4 & Make a kiss (lips forward) \\
E5 & Show the upper row of teeth \\
E6 & Elevate the eyebrows \\
E7 & Strongly close the eyes \\
E8 & Normally close the eyes \\
E9 & Sentence: "Une avenue illumine borde d'arbres fleuris"
\end{tabular}

RGB images of size $1920 x 1080$. Similarly to the paralyzed patients, we collected 72 sequences of the healthy subjects and applied the same temporal normalization to obtain a total number of 3380 images of healthy subjects.

\section{B. Results and discussions}

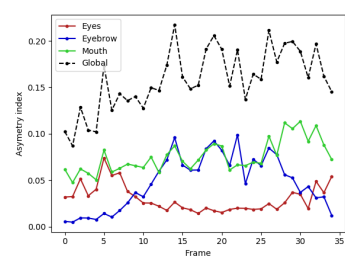

(a) Before injections

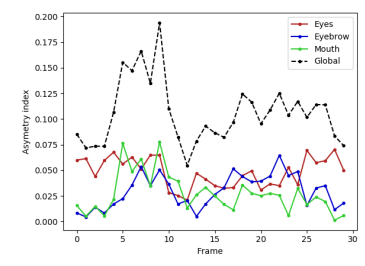

(b) After injections
Fig. 2: Global and local asymmetry graphs of patient P17 for expression E4. The black dashed curves denote the global asymmetry graphs for the whole face. The red, blue, and green curves represent the eye, eyebrow, and mouth region, respectively. Best viewed in color.

Qualitative evaluation- As a first step, in Fig. 2 we show the obtained results for a selected patient (P17) conveying the facial expression E4 (i.e., make a kiss). The plotted curves in this figure correspond to the temporal evolution of the asymmetry indices computed globally and locally along the video before (left panel) and after (right panel) the treatments. We can observe that the provided global asymmetry indices (black dashed curve) before treatments (left) are higher than those after treatments (right). This means that the treatment improved the facial symmetry of the patient in this expression which is in accordance with the clinical observation. Moreover, during this expression (E4), the patient have to make a kiss, hence only the mouth region is expected to move. However, we can observe in the left panel of Fig. 2 a peak on the blue curve denoting the local asymmetry indices for the region of eyebrows before the treatments. This peak corresponds to an involuntary eyebrow elevation when the patient tried to put his lips forward due to facial paralysis. This means that, as claimed in section II-C,

TABLE II: Comparison of the asymmetry indices computed for the patients before and after treatments and the healthy subjects

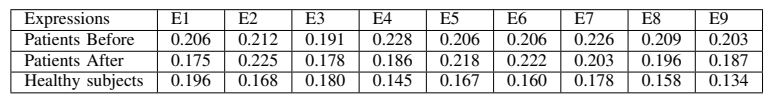


TABLE III: Detailed comparison of our results with respect to the medical scales

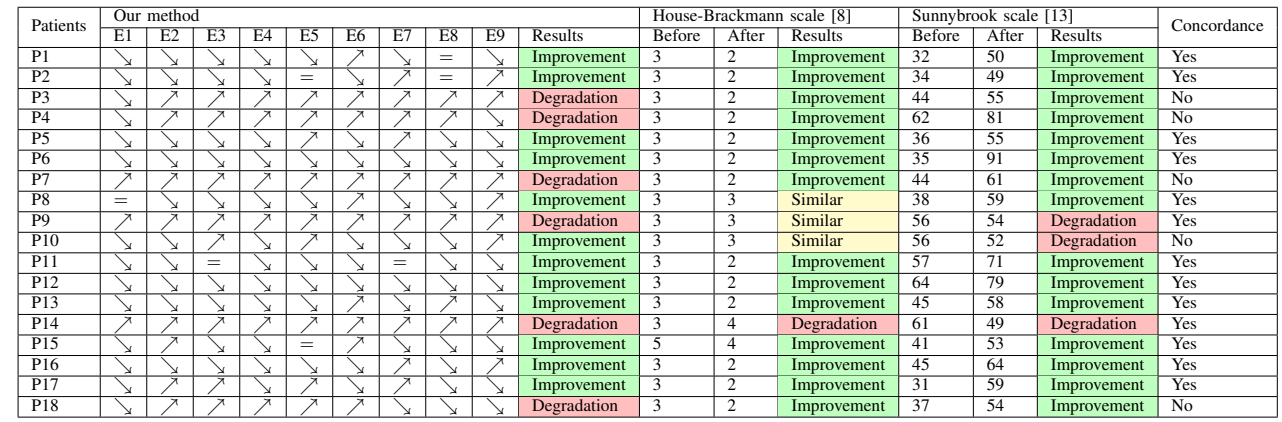

our approach can detect these involuntary facial movements. On the graph after the treatments, the blue curve is more stable than before indicating an improvement in the eyebrow region for this patient.

Quantitative evaluation- In order to quantitatively evaluate the proposed method in the assessment of facial asymmetry, we applied it on three different subsets from the collected dataset which are the healthy subjects and the patients before and after treatments. Here, we are expecting higher values of facial asymmetry indices for patients before treatment and lower values for patients after treatment and healthy subjects. To compare the asymmetry indices across the three subsets, we start by computing the mean of the asymmetry indices of each video to obtain a single asymmetry index per video. Then, for each expression we take the median value of the mean asymmetry indices computed on the different subjects conveying this expression. By doing so, we obtain a single asymmetry index for each expression in each subset as summarized in Table II. In this Table, we can observe that the asymmetry indices for healthy subjects are notably lower than those computed for patients. This means that the healthy subjects had more symmetrical faces than the patients before and after treatment. One can also note that for six expressions from nine (E1, E3, E4, E7, E8 and E9), the asymmetry indices for the patients after the treatments are lower than the ones before indicating a global improvement of the facial symmetry for the patients after the treatments.

As a last analysis, we evaluate the reliability of the automatically measured asymmetry indices in indicating the improvement or deterioration of facial paralysis with respect to clinical annotations. On one hand, as stated in the previous section, each recorded session (i.e., collection of nine video sequences of a patient) was annotated by clinicians according to two medical scales [8], [13]. These annotations are considered as ground truth labels for each session. Hence, we can check for each patient whether there is improvement or degradation by comparing the clinical annotations of the sessions before and after treatment. On the other hand, our method allows to compute an asymmetry index for each frame of a video sequence as described in section II. To compare our results with the clinical annotations provided for each session, we start by computing the mean of the asymmetry indices of each video to obtain a single asymme- try index per video (i.e., expression). Then, for each patient we check the improvement in the different expressions by comparing the mean asymmetry indices of each expression before and after treatment. To decide whether there is a global improvement or not between the two sessions of a patient (i.e., before and after treatment), we proceed by a majority voting of the decisions provided for the different expressions. Table III summarizes the results of our method and those obtained based on the two medical scales. With the House-Brackmann scale [8], an improvement is recorded for a patient when the score given by the clinician after the treatment is lower than the one before. In contrast, with the Sunnybrook scale [13], an improvement is recorded if the score provided by the clinician after the treatment is higher than the one before. For what concerns our method, we show the detailed results for each expression. An expression is marked improved (represented by $\searrow$ in the Table) if the mean of the asymmetry indices in the video after the injections is lower than the mean of the indices before. Otherwise, the expression is marked degraded (represented by $\nearrow$ in the Table). If there is no significant changes, the expression is marked similar (represented by $=$ in the Table). From Table III, we observe that the results given by the two medical scales are consistent. They both show 15 improvements and the disagreements concerned only three patients (P8, P9, and P10). Notably, we recorded with our automatic method 12 improvements and 6 degradations. The last column of the table indicates if there is a concordance between our results and those provided by the medical scales. We observe that our results are in accordance with the clinical annotations for 13 patients from 18, which represents $\mathbf{7 2 \%}$ of concordance.

\section{CONCLUSIONS AND FUTURE WORKS}

We have developed a novel technique to quantify objectively the asymmetry of the face and we tested it on a new collected dataset. Our technique is based on the natural symmetry of the human face and uses the barycentric coordinates of 2D tracked landmarks to compute the asymmetry index. We demonstrate that our technique gives promising results by comparing it with the medical scales. In the future, we will evaluate the reproductibility and the repeatability of the experimental results under different acquisition conditions. 


\section{REFERENCES}

[1] R. Clark and C. Berris. Botulinum toxin: a treatment for facial asymmetry caused by facial nerve paralysis. Plast Reconstr Surg, 84(2):3535, 1989.

[2] P. A. Desrosiers, Y. Bennis, M. Daoudi, B. Ben Amor, and P. Guerreschi. Analyzing of facial paralysis by shape analysis of $3 \mathrm{~d}$ face sequences. Image Vision Comput., 67:67-88, 2017.

[3] R. Filipo, I. Spahiu, E. Covelli, M. Nicastri, and G. Bertoli. Botulinum toxin in the treatment of facial synkinesis and hyperkinesis. The Laryngoscope, 122(2):266-70, 2012.

[4] A. Gaber, M. F. Faher, and M. A. Waned. Automated grading of facial paralysis using the kinect v2: A proof of concept study. In 2015 International Conference on Virtual Rehabilitation (ICVR), pages 258-264, 2015.

[5] A. Gaber, M. F. Taher, and M. A. Wahed. Quantifying facial paralysis using the kinect v2. In EMBC, pages 2497-2501. IEEE, 2015.

[6] A. Gerós, R. Horta, and P. Aguiar. Facegram - objective quantitative analysis in facial reconstructive surgery. Journal of Biomedical Informatics, 61:1-9, 2016

[7] R. Horta, P. Aguiar, D. Monteiro, A. Silva, and J. M. Amarante. A facegram for spatial-temporal analysis of facial excursion: Applicability in the microsurgical reanimation of long-standing paralysis and pretransplantation. Journal of Cranio-Maxillofacial Surgery, 42(7):1250-1259, 2014.

[8] J. W. House and D. E. Brackmann. Facial nerve grading system. OtolaryngologyHead and Neck Surgery, 93(2):146-147, 1985.

[9] A. Kacem, M. Daoudi, and J. C. Álvarez Paiva. Barycentric representation and metric learning for facial expression recognition. In $F G$, pages 443-447. IEEE Computer Society, 2018.

[10] A. Kacem, Z. Hammal, M. Daoudi, and J. Cohn. Detecting depression severity by interpretable representations of motion dynamics. In Automatic Face \& Gesture Recognition (FG 2018), 2018 13th IEEE International Conference on, pages 739-745. IEEE, 2018.

[11] H. S. Kim, S. Y. Kim, Y. H. Kim, and K. S. Park. A smartphonebased automatic diagnosis system for facial nerve palsy. Sensors, 15(10):26756-26768, 2015.

[12] F. D. la Torre, W. Chu, X. Xiong, F. Vicente, X. Ding, and J. F. Cohn. Intraface. In $F G$, pages 1-8. IEEE Computer Society, 2015.

[13] B. G. Ross, D. G. Fradet, and D. J. M. Nedzelski. Development of a sensitive clinical facial grading system. OtolaryngologyHead and Neck Surgery, 114(3):380-386, 1996. 